\title{
Erratum to: Derivation of tunneling probabilities for arbitrarily graded potential barriers using modified Airy functions
}

\author{
Kyu-Tae Lee • Eun Joo Jung • Chul Han Kim • \\ Chang-Min Kim
}

Published online: 25 October 2013

(C) Springer Science+Business Media New York 2013

\section{Erratum to: Opt Quant Electron (2010) 42:129-141 DOI 10.1007/s11082-010-9434-7}

The authors would like to rectify the incorrect equation, figure, and relevant comments in the above publication.

Erratum I (p. 133)

The tunneling probability is then calculated to be

$$
T_{M A F}=\left|\frac{c_{7}}{c_{1}}\right|^{2}=\left|\frac{j 2 q_{0} \Delta_{1} \Delta_{2}}{\widetilde{A}_{1} B_{T}+A_{T} \widetilde{B}_{1}}\right|^{2}
$$

Erratum II (p. 134)

As shown in Fig. 3, the MAF method shows fairly low values in the entire range of E, which is, in low-energy range, in agreement with the previous reports by Roy et al. (1993), Kimeu et al. (2008). It is also seen that the MAF results fall to 0 as $E / V_{0}$ approaches 1 , which is

The online version of the original article can be found under doi:10.1007/s11082-010-9434-7.

K.-T. Lee · C. H. Kim · C.-M. Kim ( $\varangle)$

School of Electrical and Computer Engineering, University of Seoul, 13 Siripdae-gil,

Dongdaemun-gu, Seoul, Korea

e-mail: cmkim@uos.ac.kr

K.-T. Lee

e-mail: photon.ktlee@gmail.com

C. H. Kim

e-mail: chkim@uos.ac.kr

E. J. Jung

Nano-Photonics Research Center, Korea Photonics Technology Institute, Gwangju 500-799, Korea e-mail: fstock@kopti.re.kr 
Fig. 3 Tunneling probabilities for inverse hyperbolic cosine potential barrier of Fig. 2.

( $x_{0}:$ midpoint $)$

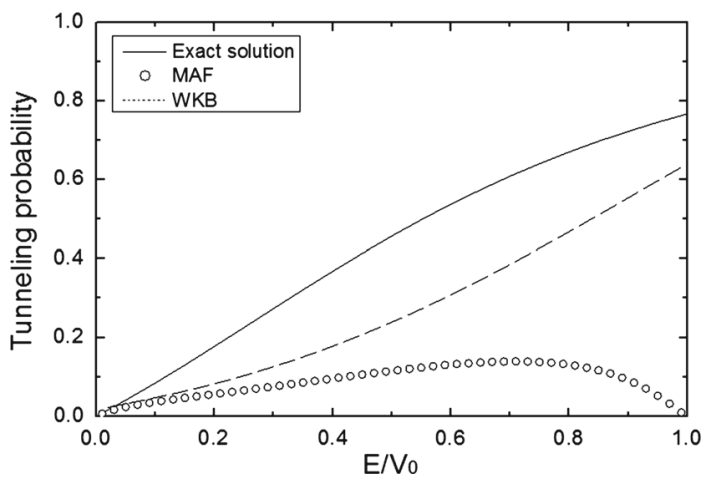

presumed to be attributed to the fact that there is a point where $V^{\prime}(x)=0$ within the potential profile, a midpoint for this bell-shaped case.

Erratum III (p. 138)

\section{Conclusion}

Three types of potential barriers, a bell-shaped barrier, a symmetric exponential barrier, and a truncated exponential barrier, were taken as sample cases to validate the accuracy of the proposed method. It turned out that the MAF method yielded very accurate results for latter two cases, while the method failed for the bell-shaped case. It appeared that the MAF approach is not suitable for graded potential barriers where the potential profile included a point or points where $V^{\prime}(x)=0$ within the region of analysis as in Fig. 2.

\section{References}

Kimeu, J., Mai, R., Majumdar, K.: Application of the variational R-matrix method to one-dimensional quantum tunneling. arXiv:quant-ph/0407249v1 (2008)

Roy, S., Ghatak, A.K., Goyal, I.C., Gallawa, R.L.: Modified airy function method for the analysis of tunneling problems in optical waveguides and quantum-well structures. IEEE J. Quantum Electron. 29, 340-345 (1993) 